\title{
Research on Airborne Near Infrared Spectroscopy Equipment
}

\author{
Chunyu Wang, Yang Li, Qingwei Dong, Yingjie Sun, \\ Yunfeng Liang \\ Department of Aircraft Control \\ Aviation University of Air Force \\ Changchun, 130022, China; \\ e-mail: sure050707@,foxmail.com
}

\author{
Peng Tian \\ Department of Aircraft Control \\ Aviation University of Air Force \\ Changchun, 130022, China \\ e-mail: sure050707@,foxmail.com
}

\begin{abstract}
After the experiment at near darkroom conditions, using sodium light through the iris diaphragm to the Fourier spectrometer, the spectrometer is completed by adjusting the interference of sodium, Fourier transform, the peak value of the interference, is received by the detector to the computer. After the image acquisition software acquisition and storage, and then the error analysis and processing, in order to verify the measurement accuracy of the spectrometer can reach 1nm.During the experiment, in order to weaken the influence of stray light on the measurement of the interferogram, and the SNR, we used a black box approach, on the black cloth covered multilayer experimental platform, the stray light offset further validate the reliability of the experimental system.
\end{abstract}

Keywords-near infrared spectroscopy; spectral transformation; fourier.

\section{INTRODUCTION}

In recently years, near-Infrared Spectroscopy (NIR) is the more rapid development of high-tech analysis technology. It has the fastly, efficient, non- loss and adapt to analysis online and many other advantages, so that in agriculture, pharmaceutical, petrochemical and military imaging or other fields has been widely used. Near infrared spectroscopy is an important feature to achieve a near-infrared spectrometer, analytical models and chemometrics software integration. NIR is the basis and premise of the technology is its performance is stable and reliable.

\section{NIR IMAGING ON MILITARY APPLICATIONS PROFILE}

Because of the great advantages of high spectral resolution imaging spectrometer in space Earth observation while obtaining numerous feature continuous band spectral image recognition reach the Earth's surface from space directly substances aim to become a hot topic in the field of remote sensing, it is becoming the main technical means of a contemporary space observe on the ground. Therefore, about unmanned aerial vehicles (UAV) and airborne hyperspectral imaging to measure field, the near-infrared spectral imaging has a wide range of applications. The currently airborne hyperspectral imager of its small size, light weight, easy installation, high optical performance and stability, spectral coverage 400-
$1000 \mathrm{~nm}$, very suitable for installation on unmanned aerial vehicles and small aircraft applications.

\section{FOURIER TRANSFORM SPECTROMETER}

\section{A. Fourier transform spectrometer principle and composition.}

Fourier transform spectrometer is adopt the double beam interference principle, the phase difference between the beam of coherent changes continuously, and recorded synchronously the light intensity changes curve interferogram of central stripe. Then it is subjected to Fourier transform to obtain a light spectrum figure.

Using a frequency modulation method, so that the modulated light of different wavelengths in different frequency, modulated by a Fourier integral transform to obtain spectral information at different wavelengths. Fourier transform spectrometer is generally composed with a light source, interferometer, the receiver and the computing system and the output system. At Present, Michelson interferometer is used to achieve interference FM .The Fig.1 shows that Michelson interferometer FM Fourier transform spectrometer compositions and working process. It reflects the principle of Fourier transform spectrometer[1].

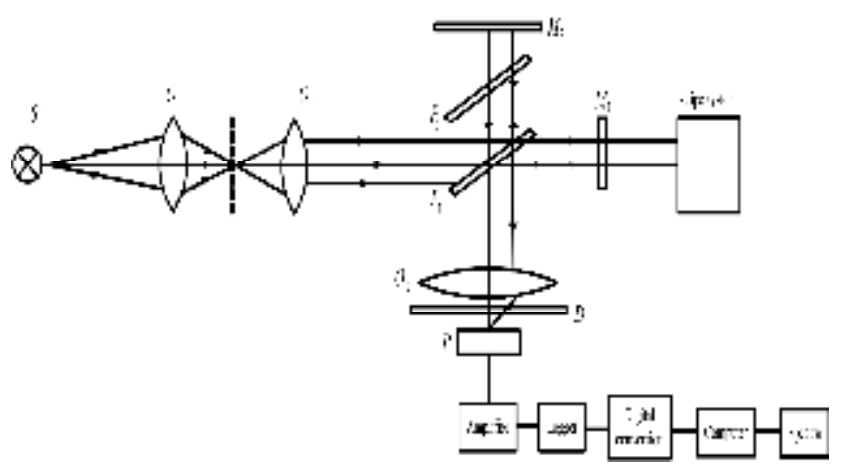

Fig.1: michelson interferometer FM Fourier transform spectrometer principle schematic

The radiation emitted by the light source $\mathrm{S}$, after being condensed by condenser illumination into perforation $\mathrm{O}, \mathrm{O}_{1}$ by the collimating lens into a parallel beam onto a splitter plate $\mathrm{P}_{1}$. Splitting the incident beam into the plate $\mathrm{P}_{1}$ equal to two coherent light beams; beam directed through the splitter plates $P_{1}$ movable plane mirror $M_{1}, M_{1}$ and is reflected back to the splitter plate $\mathrm{P}_{1}$; Another 
bunch of reflected light directed through the compensating plate $\mathrm{P}_{2}$ after the plane mirror $\mathrm{M}_{2}, \mathrm{M}_{2}$, was reflected to the splitter plates $\mathrm{P}_{1}$, the two met again and the light beams interfere. When the flat mirror $\mathrm{M}_{1}$ is moved, the optical path difference between the two beams is changed.The interferogram recording function and converting the optical path difference of stripe system, and then fed into a computer system, by computing output the spectrum[2].

Set $\bar{v}$ wave number that two monochromatic beams interfere, and to form an interference image intensity distribution $I^{\prime}(x), x$ is the optical path difference, so it can get

$$
I^{\prime}(x)=E(\bar{v})(1+\cos 2 \pi \bar{v} x)
$$

Where $E(\bar{v})$ is the spectral intensity of indexing wave number $\bar{v}$.

If the wave number of the light source and the light beam incident on the interferometer when $(\bar{v}, \bar{v}+d \bar{v})$, Interferometric images obtained distribution:

$$
I^{\prime}(x)=\int_{\bar{v}}^{\bar{v}+d \bar{v}} E(\bar{v})(1+\cos 2 \pi \bar{v} x) d \bar{v}
$$

Therefore, the total flux is:

$$
I^{\prime}(x)=\int_{0}^{\infty} E(\bar{v}) d \bar{v}+\int_{0}^{\infty} E(\bar{v}) \cos 2 \pi \bar{v} x d \bar{v}
$$

The first one is on the right-hand side and the optical path difference and other changes unrelated large items. When the optical path difference is zero, is $x=0$ :

$$
I^{\prime}(x)=I^{\prime}(0)=2 \int_{0}^{\infty} E(\bar{v}) d \bar{v}
$$

When the optical path difference is $D$,

$$
I^{\prime}(D)=\frac{1}{2} I(0)+\int_{0}^{\infty} E(\bar{v}) \cos 2 \pi \bar{v} D d \bar{\nu}
$$

The recording system records only the receiver portion of the image of the interference variable, So the actual function of the resulting interference pattern:

$$
I^{\prime}(x)=\int_{0}^{\infty} E(\bar{v}) \cos 2 \pi \bar{v} D d \bar{v}
$$

Since the Fourier transform reversible, so the interference pattern function $I(x)$ can be calculated spectrum intensity distribution of the light source

$$
E_{(\bar{v})}=\int_{0}^{\infty} I_{(x)} \cos 2 \pi \bar{v} x d x
$$

Continuously changing the optical path difference of the interferometer, Photoelectric element interferometer can record variable optical radiation emitted flux, Get "interferogram function." Interferogram as Fourier cosine transform, you can get the radiation intensity of any wave number[3].

\section{B. Fourier transform spectrometer resolving ability.}

According to the basic equations for the Fourier transform shows that the optical path difference integral infinite, but actually attainable optical path difference is always limited. Therefore, the actual spectrum obtained by Fourier transform of the spectrum produced compared to deviate. It's a function of the interference pattern depends on the instrument ideal monochromatic interference pattern generated Fourier transform function graph. If the device can achieve a maximum interference optical path difference is $\pm x_{m}$, after transform interference pattern of the ideal monochromatic $E(\bar{v})$, what obtained can be approximated by the following graphic formula

$A\left(\bar{v}-\bar{v}_{0}\right)=\frac{\sin \left[2 \pi\left(\bar{v}-\bar{v}_{0}\right) x_{m}\right]}{2 \pi\left(\bar{v}-\bar{v}_{0}\right) x_{m}}$

$A\left(\bar{v}-\bar{v}_{0}\right)$ called the instrument function. Two zero value near the center of the

$$
\bar{v}-\bar{v}_{0}= \pm \frac{1}{2 x_{m}}
$$

Among them, the half-intensity width

$$
\delta \bar{v}=\bar{v}-\bar{v}_{0}=\frac{1}{2 x_{m}}
$$

(10) Show that the resolution of the Fourier transform spectrometer with the maximum optical path difference $x_{m}$ of two interfering light beams is inversely proportional. Therefore the greater the distance of movement of the movable mirror, $\delta \bar{v}$ the smaller, the higher the resolution.

However, in the above cases when the input beam is collimated beam of ideal conditions the conclusion that it is required a light source to be an ideal point source. In the actual apparatus, the light source is always a certain size, but that is not a point light source but extended source, the solid angle of the instrument with a sheet size $\Omega$. When using monochromatic extended source, the wave number $\bar{v}_{0}$ of Monochrome spectral line through interferometer that will becomes narrow by a rectangular spectral band, which extend the range of wave number

$$
\delta \bar{v}=\bar{v}_{0} \frac{\Omega}{2 x_{m}}
$$

The expansion of the wave number value determines the Fourier transform spectrometer may reach the limit of resolution. Therefore, in actual instrument, we can not indefinitely increase the maximum optical path difference $x$ to achieve arbitrarily high resolution[4].

\section{THE CHARACTERISTICS OF THE FOURIER TRANSFORM SPECTROMETER}

Fourier transform spectrometer have different characteristics.

i) Rapid analysis. Due to the spectral measurement process can generally be completed within 1min (multichannel instrument can be completed within $1 \mathrm{Sec}$ ), by correcting the model can be quickly determined the composition or properties of the sample.

ii)Analysis of high efficiency. Through a measured spectrum and the corresponding calibration model has been established, or simultaneously a plurality of properties of the sample composition were measured [5]. 
iii) Low test cost. Near infrared spectroscopy in the analysis process does not consume the sample, in addition to their own little electricity consumption has almost no other consumption, compared with the commonly used standard or reference method, the test costs can be significantly reduced.

iv) Reproducible test. Due to the stability of the spectral measurements, the test results is less affected by human factors, compared with the standard or reference method, near infrared spectroscopy generally showed the better reproducibility.

v) Needless sample pretreatment measurements are generally convenient spectral measurements. Penetration and scattering effects due to the near-infrared light is strong, according to the strength of the sample and the light transmission capability of state can be used to transmit or diffuse reflectance spectra measured way. Specimen measurement device through the corresponding samples of different physical state of a liquid, solid, semisolid and jelly etc. can be measured directly.

vi) Easy to implement on-line analysis. Since the nearinfrared light good transmission characteristics in the optical fiber, the optical fiber by the instrument away from the sampling site may be, the measured spectrum signal transmitted in real time to the instrument, after the establishment of the call calculated calibration model can be displayed directly in the composition of the sample or production means the nature of the results. Further, the optical fiber can also be measured by the harsh environment of the sample.

vii)Typical non-destructive analysis techniques. Spectral measurement process does not consume the sample, the sample will not have an impact from the exterior to the internal. Given this characteristic, the technology in the field of medicine and clinical analysis in vivo are being more and more applications[6].

\section{EXPERIMENT AND EXPERIMENTAL ANALYSIS}

Instruments and equipment required for the experiment include: Test source (sodium), the Fourier transform spectrometer, and computer. In determining the spectrometer components parameters, determined in accordance with the above principles and experimental methods to experiment, collecting experimental data in interference image.

Test light from the source via a variable diaphragm to Fourier spectrometer, Interference within the spectrometer, and then received by the detector and sent the computer. Experimental data are derived from the measurement of the device by the image acquisition software acquisition and storage, and then analysis and processing[7].

After experiment,when sodium light intensity is stabled, adjust its height to let light through spectral diaphragm, and adjust the signal gain that can be obtained experimentally measured light intensity metering image. The Fig. 2 shows that by adjusting the spectrometer to obtain sodium interferogram. The Fig. 3 shows that After the Fourier transform, we'll get the image of the wave peak interference.

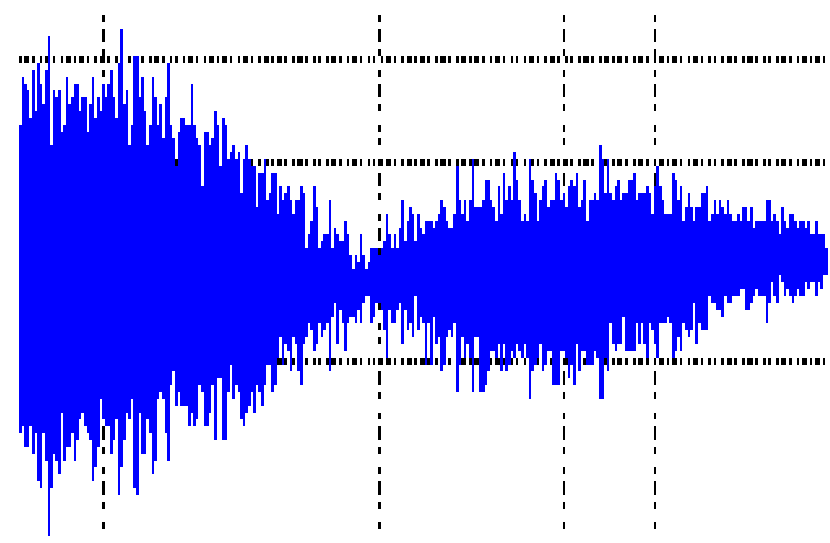

Fig.2: sodium interference

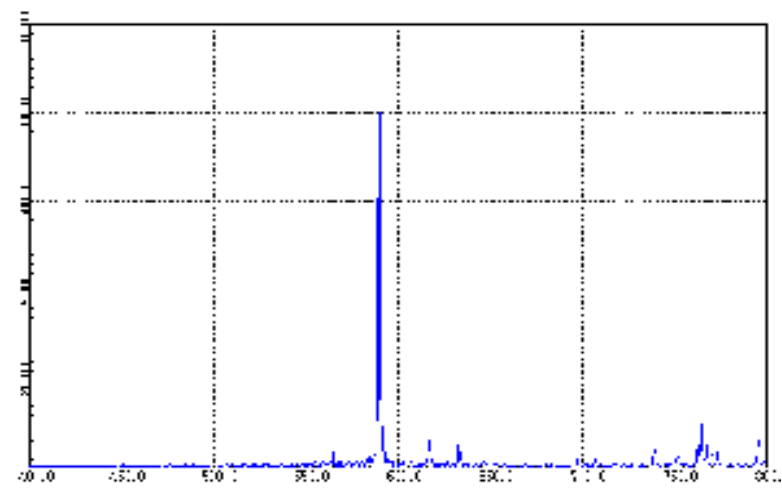

Fig.3: Sodium interferogram obtained by Fourier transform spectra

Experimental Data

Table 1: sodium wave peak (units:nm)

\begin{tabular}{llllllll}
589.6 & 630.1 & 632.8 & 639.9 & & & & \\
589.8 & 591.4 & 630.1 & 633.0 & 664.2 & 636.8 & & \\
587.8 & 589.6 & 626.6 & 630.1 & 631.1 & 632.8 & 636.9 & 664.4 \\
\hline
\end{tabular}

We know that the wavelength of sodium is 589.0 、 589.6 . Table 1 shows that: the measured data contains $589.6,632.8$ and 636.9. Where in the wavelength of sodium light is 589.6, and 632.8 is the He-Ne laser's wavelength, 636.9 is the natural light's wavelength, while other data is the effect of natural light (the range of natural light's wavelength is $390 \sim 780 \mathrm{~nm}$ ) [8]. 
Table 2: Spectrum Analysis

\begin{tabular}{lllllll}
\hline Light source & $\begin{array}{l}\text { wavelength measured } \\
\text { value(nm) }\end{array}$ & $\begin{array}{l}\text { Average } \\
(\mathrm{nm})\end{array}$ & $\begin{array}{l}\text { Actual } \\
\text { Wavelengt } \\
\mathrm{h}(\mathrm{nm})\end{array}$ & $\begin{array}{l}\text { Margin of } \\
\text { error (\%) }\end{array}$ \\
\hline Sodium lamp & 589.6 & 589.8 & 589.6 & 589.67 & 589.6 & $0.01 \%$ \\
He-Ne Laser & 632.8 & 630.0 & 632.8 & 632.87 & 632.8 & $0.01 \%$ \\
\hline
\end{tabular}

ii)Study the SNR of equipment; enhance the

Table 2 shows that the experimental value Within the scope of it's error measurement. At the same time, the spectrum of the instrument's measurement accuracy can reach $1 \mathrm{~nm}$.

Affect the experimental data including that: the sodium unstable voltage affect the experimental data;in the experiments, the small vibration of experimental platform can affect the experimental data.For this factor,we can increase the damping measures to reduce its effects[9].

In this experiment, the stray light and the effect of the interferometric measurement system of FIG especially SNR. Although the experiments were done in the past darkroom conditions, but there are still a lot of image noise of stray light, so we use the improved experimental black-box approach, multi-layer black cloth covering on the experimental platform, played a very good stray light offset effect[10].

\section{CONCLUSIONS}

Through experiments we have concluded that:

1.Equipment matching errors, processing errors.etc, you need to experiment to be eliminated through repeated testing.

2.For optical systems, optical aberrations, we also need to be considered, including the impact of an optical telescope, sighting system will affect the quality of the acquisition and interference spatial resolution.

3. Since there are more factors about equipment conditions and involve subject and technology elements, there are still so much work to do on this experiment.

i)Improve the accuracy of experimental equipment; reduce the exterior light impacts the experimental data.

performance of the instrument on this basis.

iii)Study further image processing algorithm, improve the existing ones, to make following research.

\section{ACKNOWLEDGEMENTS}

This work was financially supported by My tutor Changchun University of Technology of the associate professor Zhao Zhenming's technical support in the academic and experimental testing.

\section{REFERENCES}

[1] Weng Shi-fu, Fourier transform infrared spectroscopy (second edition). Beijing: Chemical Industry Press, 2010.

[2] Lu Wan-zhen, Modern Near Infrared Spectroscopy (second edition), Beijing: China Petrochemical Press, 2006.

[3] Xu Guang-Tong, Yuan Hong-fu, Lu Wan-zhen, Modern Near Infrared Spectroscopy and Its Application Progress. Spectroscopy and Spectral Analysis, 20(2), pp.134-142, April 2000.

[4] Ying Ding, infrared spectroscopy instrumentation Research Progress and Development Trend. Infrared, 33(7), pp.1-5, July 2012.

[5] Xu Guang-Tong, Yuan Hong-fu, Lu Wan-zhen, Near infrared spectroscopy analysis and application. Eucalypt Science and Technology, 29(1), pp. 49-54, April 2012.

[6] Chu Xiao-Li, Yuan Hong-fu, Near infrared spectroscopy technology development and application status. Spectroscopy and Spectral Analysis, 17(5), pp. 1-4, September 2011.

[7] Xiang Li-bin,Ji Zhong-ying,Huang Hao,Spectral imager calibration technology research spatially modulated interference. Acta Photonica Sinica, 7 (4), pp. 83-86, July2004.

[8] Yan YL. Foundation and Application of Near Infrared Spectroscopy, Beijing: China Light Industry Press, 2005.

[9] Lu Wan-zhen,Yuan Hong-fu,Xu Guang-tong. Modern Near Infrared Spectroscopy, Beijing: China Petrifaction Press, 2000.

[10] J Lu, W. F. McClure, F. E.Barton, D.S.Himmelsbach. Near Infrared Spectroscopy, pp. 77, June1998. 